\title{
Self-boundedness and self-hiddenness for implicit two-dimensional systems
}

\author{
Lorenzo Ntogramatzidis
}

\begin{abstract}
In this paper we introduce and develop the concepts of self-boundedness and self-hiddenness for implicit twodimensional systems. The aim of this note is to show that when extending such concepts to a multidimensional setting, a richer structure arises than in the one-dimensional case.
\end{abstract}

\section{INTRODUCTION}

In the last forty years, geometric control has played a fundamental role in understanding several structural properties of both linear and nonlinear systems and in the solution of important control/estimation problems. The interested readers are directed to the comprehensive monographs [21], [2], [20]. In more recent times, several efforts have been devoted to the adaptation of the classic geometric approach to implicit (or singular, or descriptor) systems [3]. An important adaptation of geometric techniques was proposed in [4], which identified for the first time a definition of controlled invariance for the explicit first-order 2-D Fornasini-Marchesini model [9]. Further developments in this multidimensional setting were presented in [10] and [18]. In recent years, in [14] a new geometric setting was established for an explicit 2-D local state model that is sufficiently general to realise any quarter-plane causal bivariate transfer function, but that enables the solutions of the local state model to be characterised in terms of necessary and sufficient conditions. In [14] the notion of self-boundedness was also generalised for the first time to regular 2-D systems. In that paper, it was found that, as in the 1-D case, the set of 2-D selfbounded subspaces forms a lattice, with respect to subspace addition and intersection, and therefore admits a minimum and a maximum.

The approach developed in [14] was partially extended to singular models in [15]. In particular, in [14] the notion of controlled invariance of feedback type is generalised to 2-D systems in descriptor form. However, to date no results have been obtained on the notion of self-boundedness for two-dimensional systems in implicit form. The aim of this paper is to fill this gap, by providing a system-theoretic definition of self-boundedness which follows the same line of argument used in [13]. Then, a geometric characterisation of self-boundedness is introduced, which is shown to be intrinsically richer than the one which is valid for regular systems. We also provide an algorithm for the computation of the minimum of the lattice of self-bounded subspaces.

This work was supported in part by the Australian Research Council under the grant FT120100604.

L. Ntogramatzidis is with the Department of Mathematics and Statistics, Curtin University, Perth WA 6845, Australia. L.Ntogramatzidis@curtin.edu.au
By duality, in the last part of this paper we also define the concept of self-hiddenness.

Notation. Throughout, we denote by $\mathbb{Z}$ and $\mathbb{N}$ the integers and the natural numbers, respectively. The image and nullspace of a linear operator $M$ are denoted by $\operatorname{im} M$ and $\operatorname{ker} M$, respectively. The Moore-Penrose pseudo-inverse of $M$ is denoted with $M^{\dagger}$. Given $A: \mathbb{R}^{n} \rightarrow \mathbb{R}^{m}$ and a subspace $\mathscr{Y} \subseteq \mathbb{R}^{m}$, we denote by $A^{-1} \mathscr{Y}$ the set $\left\{x \in \mathbb{R}^{n} \mid A x \in \mathscr{Y}\right\}$. Subspaces are denoted by calligraphic letters. The annihilator of a subspace $\mathscr{S}$ of the linear space $\mathscr{X}$ is denoted by $\mathscr{S}^{\perp}$, and resides in the dual space of $\mathscr{X}$.

\section{InVARIANT SubSPACES FOR Singular FM MODELS}

Consider the implicit Fornasini-Marchesini (FM) 2-D model [8], [7]

$$
\begin{aligned}
E x_{i+1, j+1} & =A_{0} x_{i, j}+A_{1} x_{i+1, j}+A_{2} x_{i, j+1}+B u_{i, j}, \\
y_{i, j} & =C x_{i, j}+D u_{i, j}
\end{aligned}
$$

where, for all $i, j \in \mathbb{Z}$, the vector $x_{i, j} \in \mathscr{X}=\mathbb{R}^{n}$ is the partial local state, $u_{i, j} \in \mathscr{U}=\mathbb{R}^{m}$ is the input, $y_{i, j} \in \mathscr{Y}=\mathbb{R}^{p}$ is the output. Hence, $E, A_{0}, A_{1}, A_{2} \in \mathbb{R}^{q \times n}, B \in \mathbb{R}^{q \times m}, C \in \mathbb{R}^{p \times n}$ and $D \in \mathbb{R}^{p \times m}$. The outer state-space, corresponding to the number of equations in (1), is denoted by $\underline{\mathscr{X}}=\mathbb{R}^{q}$. In this implicit model the matrices $E, A_{0}, A_{1}, A_{2}$ are in general not square, and if they are square (i.e., if $q=n$ ), they may be singular. For the sake of brevity, we identify the system (1-2) with the septuple $\Sigma \stackrel{\text { def }}{=}\left(E ; A_{0}, A_{1}, A_{2} ; B ; C ; D\right)$.

We are interested in the evolution of 'south-west' causal solutions [17] given suitable boundary conditions $x_{i, j}$ for $(i, j) \in \mathfrak{B}_{0}$, where

$\mathfrak{B}_{k} \stackrel{\text { def }}{=}(\{k\} \times\{h \in \mathbb{Z} \mid h \geq k\}) \cup(\{h \in \mathbb{Z} \mid h \geq k\} \times\{k\}), \quad k \in \mathbb{Z}$. Within this setting, the latent variable evolves over the region $\mathfrak{B} \stackrel{\text { def }}{=} \bigcup_{k=0}^{\infty} \mathfrak{B}_{k}=\{(i, j) \in \mathbb{Z} \times \mathbb{Z} \mid i \geq 0$ and $j \geq 0\}=\mathbb{N} \times \mathbb{N}$.

Given a subspace $\mathscr{S}$ of $\mathscr{X}$, we say that (1) has an $\mathscr{S}$ valued boundary condition if there exists a solution of (1) with $x_{i, j} \in \mathscr{S}$ for all $(i, j) \in \mathfrak{B}_{0}$. We also say that (1) has an $\mathscr{S}$-valued solution if there exists a solution of (1) with $x_{i, j} \in \mathscr{S}$ for all $(i, j) \in \mathfrak{B}$.

We recall that a subspace $\mathscr{J} \subseteq \mathscr{X}$ is called invariant for $\left(E ; A_{0}, A_{1}, A_{2}\right)$ if

$$
A_{i} \mathscr{J} \subseteq E \mathscr{J}
$$

for all $\in\{0,1,2\}$, [15], [16]. Invariant subspaces are useful tools in the investigation of the so-called compatible 
boundary conditions of (1), i.e., the boundary conditions $\left\{x_{i, j} \in \mathscr{X} \mid(i, j) \in \mathfrak{B}_{0}\right\}$ for which an admissible solution $\left\{x_{i, j} \in \mathscr{X} \mid(i, j) \in \mathfrak{B}\right\}$ exists with $u_{i, j}=0$ for all $(i, j) \in \mathfrak{B}$.

The following lemma [15], [16] shows the relation between the concept of invariance for $\left(E ; A_{0}, A_{1}, A_{2}\right)$ defined here and the existence of compatible solutions for $\Sigma$.

Lemma 1: [15], [16]. Subspace $\mathscr{J}$ of $\mathscr{X}$ is invariant for $\left(E ; A_{0}, A_{1}, A_{2}\right)$ if and only if (1) has a solution $\left\{x_{i, j} \in\right.$ $\mathscr{J} \mid(i, j) \in \mathfrak{B}\}$ for any $\mathscr{J}$-valued boundary condition with zero input.

The set of invariant subspaces of $\left(E ; A_{0}, A_{1}, A_{2}\right)$ is closed under subspace addition. Therefore, it admits a maximum element, which is given by the sum of all invariant subspaces of $\left(E ; A_{0}, A_{1}, A_{2}\right)$. This subspace, herein denoted by $\mathscr{J}^{\star}$, can be computed using the following result, see [15], [16].

Lemma 2: [15], [16]. The subspace $\mathscr{J}^{\star}$ can be computed as the last term of the monotonically non-increasing sequence of subspaces $\left\{\mathscr{J}_{i}\right\}_{i \in \mathbb{N}}$ given by

$$
\left\{\begin{aligned}
\mathscr{J}_{0} & =\mathscr{X} \\
\mathscr{J}_{i} & =\bigcap_{j=0}^{2}\left(A_{j}^{-1} E \mathscr{J}_{i-1}\right) \cap \mathscr{J}_{i-1} \quad i \in\{1,2, \ldots, k\},
\end{aligned}\right.
$$

where the integer $k \leq n-1$ is determined by the condition $\mathscr{J}_{k+1}=\mathscr{J}_{k}\left(=\mathscr{J}^{\star}\right)$.

\section{CONTROLlED INVARIANCE}

We now recall the notion of (simple) controlled invariance for the implicit FM model (1).

Definition 1: [15], [16]. A subspace $\mathscr{V}$ of $\mathscr{X}$ is a controlled invariant subspace for the quintuple $\left(E ; A_{0}, A_{1}, A_{2} ; B\right)$ if

$$
\sum_{i=0}^{2}\left(A_{i} \mathscr{V}\right) \subseteq E \mathscr{V}+\operatorname{im} B
$$

Theorem 1: [15], [16]. For all $\mathscr{V}$-valued boundary conditions there exists a control input such that (1) admits a quarter-plane causal $\mathscr{V}$-valued trajectory if and only if $\mathscr{V}$ is a controlled invariant.

For the definitions of controlled invariance given in [4] and [5], where a first-order FM model was considered, only the if part of the statement of Theorem 1 holds true. Necessity for the implicit model (1) considered here holds as in the regular case of this form studied in [14].

\section{A. Output-Nulling Subspaces}

In many control problems it is of interest to derive control laws that maintain certain outputs of a system at zero. The most famous example is the disturbance decoupling problem, [2]. This requirement leads to the notion of output-nulling subspace: a subspace $\mathscr{V}$ is said to be output-nulling for $\Sigma$ if there exists a control law such that (1-2) admits a (non necessarily unique) $\mathscr{V}$-valued solution with zero output given an arbitrary $\mathscr{V}$-valued boundary condition. It is easy to see
[15], [16] that a subspace $\mathscr{V}$ is output-nulling for $\Sigma$ if and only if

$$
\left[\begin{array}{ccc}
A_{0} & A_{1} & A_{2} \\
C & O & O
\end{array}\right] \bigoplus_{k=1}^{3} \mathscr{V} \subseteq(E \mathscr{V} \oplus\{0\})+\operatorname{im}\left[\begin{array}{c}
B \\
D
\end{array}\right]
$$

where $\bigoplus_{k=1}^{3} \mathscr{V}=\mathscr{V} \oplus \mathscr{V} \oplus \mathscr{V}$

The set of output-nulling subspaces of (1-2) is denoted by $\mathscr{V}(\Sigma)$. As for the set of $2-\mathrm{D}$ controlled invariant subspaces, this set is seen to be closed under subspace addition but not under subspace intersection. Therefore, $(\mathscr{V}(\Sigma),+; \subseteq)$ is a (non-distributive and modular) upper semilattice with respect to the binary operation + and with respect to the partial ordering $\subseteq$. Thus, it admits a maximum $\mathscr{V}^{\star}$ given by the sum of all elements of $\mathscr{V}(\Sigma)$, i.e., $\mathscr{V} \stackrel{\text { def }}{=} \max \mathscr{V}(\Sigma)=\sum_{\mathscr{V} \in \mathscr{V}(\Sigma)} \mathscr{V}$. The following lemma extends the famous algorithm for the computation of $\mathscr{V}^{\star}$.

Lemma 3: [16]. $\mathscr{V}^{\star}$ is the last term of the monotonically non-increasing sequence $\left\{\mathscr{V}_{i}\right\}_{i}$ given by

$$
\left\{\begin{array}{l}
\mathscr{V}_{0}=\mathscr{X} \\
\mathscr{V}_{i}=\bigcap_{j=0}^{2}\left[\begin{array}{c}
A_{j} \\
O
\end{array}\right]^{-1}\left(\left(E \mathscr{V}_{i-1} \oplus\{0\}\right)+\mathrm{im}\left[\begin{array}{c}
B \\
D
\end{array}\right]\right) \quad i \in\{1, \ldots, k\}
\end{array}\right.
$$

where the integer $k \leq n-1$ is determined by the condition $\mathscr{V}_{k+1}=\mathscr{V}_{k}$, i.e., $\mathscr{V}^{\star}=\mathscr{V}_{k}$.

\section{B. Controlled Invariants of Feedback Type}

The FM model (1-2) is closed under the feedback $u_{i, j}=$ $F x_{i, j}$, which gives rise to the closed-loop local state update equation

$$
E x_{i+1, j+1}=\left(A_{0}+B F\right) x_{i, j}+A_{1} x_{i+1, j}+A_{2} x_{i, j+1} .
$$

Differently from 1-D systems, the notion of controlled invariance alone is not sufficient to guarantee the existence of a feedback matrix $F$ for which a solution of (1) is maintained on a controlled invariant subspace $\mathscr{V}$ for $\mathscr{V}$ valued boundary conditions. For this reason, the concept of controlled invariance of feedback type was introduced in [15]-[16]. The subspace $\mathscr{W}$ of $\mathscr{X}$ is controlled invariant of feedback type for $\Sigma$ if there exists an $F \in \mathbb{R}^{m \times n}$ such that (1), with $u_{i, j}=F x_{i, j}$, admits a $\mathscr{W}$-valued trajectory for arbitrary $\mathscr{W}$-valued boundary condition. In [15]-[16] it was shown that $\mathscr{W}$ is controlled invariant of feedback type for $\Sigma$ if and only if

- $A_{0} \mathscr{W} \subseteq E \mathscr{W}+\operatorname{im} B$

- $A_{1} \mathscr{W}+A_{2} \mathscr{W} \subseteq E \mathscr{W}$.

Given a controlled invariant subspace of feedback type $\mathscr{W}$ for $\Sigma$, any feedback matrix $F \in \mathbb{R}^{m \times n}$ such that with the input $u_{i, j}=F x_{i, j}$ the system $\Sigma$ admits a $\mathscr{W}$-valued trajectory for arbitrary $\mathscr{W}$-valued boundary condition is called friend of $\mathscr{W}$. In [15]-[16] it is shown that the set of friends of the controlled invariant subspace of feedback type $\mathscr{W}$, with basis matrix $W$, coincides with the set of matrices $F$ such that $\Omega=-F W$, where $\Omega$ is a solution of $A_{0} W=E W X_{0}+B \Omega$ 
for some matrix $X_{0}$. As such, the set of friends of $\mathscr{W}$ is parameterised as follows. First, we compute $X_{0}$ and $\Omega$ as

$$
\left[\begin{array}{c}
X_{0} \\
\Omega
\end{array}\right]=\left[\begin{array}{ll}
E W & B
\end{array}\right]^{\dagger} A_{0} W+H_{1} K_{1},
$$

where $H_{1}$ is a basis matrix for $\operatorname{ker}\left[\begin{array}{ll}E W & B\end{array}\right]$ and $K_{1}$ is an arbitrary matrix of suitable size. Then, recalling that $W$ is full column-rank, we compute

$$
F=-\Omega\left(W^{\mathrm{T}} W\right)^{-1} W^{\mathrm{T}}+K_{2} H_{2},
$$

where $\mathrm{H}_{2}$ is a full row-rank matrix such that $\operatorname{ker} H_{2} \supseteq \mathscr{W}$ (so that $H_{2} \mathrm{~W}=0$ ) and $K_{2}$ is an arbitrary matrix of suitable size. The set of friends of $\mathscr{W}$ are parameterised in this way in terms of the two matrices $K_{1}$ and $K_{2}$.

\section{Output-Nulling Subspaces of Feedback Type}

The notion of controlled invariance of feedback type can be extended to output-nulling subspaces. A subspace $\mathscr{W}$ is output-nulling of feedback type for $\Sigma$ if there exists a static local-state feedback control law $u_{i, j}=F x_{i, j}$ such that (1-2) admits a $\mathscr{W}$-valued solution for which the output is identically zero for arbitrary $\mathscr{W}$-valued boundary condition. It is easy to see [15]-[16] that $\mathscr{W}$ is output-nulling of feedback type for $\Sigma$ if and only if

- $\left[\begin{array}{c}A_{0} \\ C\end{array}\right] \mathscr{W} \subseteq(E \mathscr{W} \oplus\{0\})+\operatorname{im}\left[\begin{array}{l}B \\ D\end{array}\right]$;
- $A_{1} \mathscr{W}+A_{2} \mathscr{W} \subseteq E \mathscr{W}$.

Observe that the inclusion $\left[\begin{array}{c}A_{0} \\ C\end{array}\right] \mathscr{W} \subseteq(E \mathscr{W} \oplus\{0\})+$ $\operatorname{im}\left[\begin{array}{l}B \\ D\end{array}\right]$ is equivalent to the existence of two matrices $X_{0}$ and $\Omega$ such that

$$
\left[\begin{array}{c}
A_{0} \\
C
\end{array}\right] W=\left[\begin{array}{c}
E W \\
0
\end{array}\right] X_{0}+\left[\begin{array}{l}
B \\
D
\end{array}\right] \Omega
$$

where $W$ is a basis matrix of $\mathscr{W}$, while the inclusion $A_{1} \mathscr{W}+$ $A_{2} \mathscr{W} \subseteq E \mathscr{W}$ is equivalent to the existence of matrices $X_{1}, X_{2}$ such that $A_{i} W=E W X_{i}(i \in\{1,2\})$. Let $F$ be such that $\Omega=$ $-F W$, so that

$$
\left[\begin{array}{c}
A_{0}+B F \\
C+D F
\end{array}\right] W=\left[\begin{array}{c}
E W \\
0
\end{array}\right] X_{0} .
$$

It turns out that $\mathscr{W}$ is output-nulling of feedback type for $\Sigma$ if and only if a feedback matrix $F$ exists such that

$$
\left[\begin{array}{ccc}
A_{0}+B F & A_{1} & A_{2} \\
C+D F & 0 & 0
\end{array}\right] \bigoplus_{k=1}^{3} \mathscr{W} \subseteq E \mathscr{W} \oplus\{0\} .
$$

The next theorem addresses the problem of the uniqueness of the solution of (1) on output-nulling subspaces of feedback type, which will be instrumental for a correct identification of geometric conditions characterising self-boundedness and self-hiddenness for 2-D implicit models.

Theorem 2: Let $\mathscr{W}$ be an output-nulling subspace of feedback type, and let $F$ be a friend of $\mathscr{W}$. A solution of (1) corresponding to zero input and with zero output is the unique $\mathscr{W}$-valued solution of (1) with zero output if and only if

$$
\operatorname{ker} E \cap \mathscr{W}=\{0\}
$$

The proof follows closely the one for [15, Theorem 1].

The set of output-nulling subspaces of feedback type, denoted by $\mathscr{W}(\Sigma)$, is closed under addition (but not under intersection). Hence, the maximum output-nulling subspace $\mathscr{W}^{\star}$ of feedback type can still be defined as the sum of all the elements of $\mathscr{W}(\Sigma)$. An algorithm for the computation of $\mathscr{W}^{\star}$, that can be derived by adapting the one for $\mathscr{V}^{\star}$, is given as follows.

Lemma 4: [15]-[16]. Subspace $\mathscr{W}^{\star}$ is the last term of the monotonically non-increasing sequence of subspaces $\left\{\mathscr{W}_{i}\right\}_{i}$ given by

$$
\begin{aligned}
& \left\{\begin{array}{l}
\mathscr{W}_{0}=\mathscr{X} \\
\mathscr{W}_{i}=\left[\begin{array}{c}
A_{0} \\
C
\end{array}\right]^{-1}\left(\left(E \mathscr{W}_{i-1} \oplus\{0\}\right)+\mathrm{im}\left[\begin{array}{c}
B \\
D
\end{array}\right]\right)
\end{array}\right. \\
& \cap\left[\begin{array}{l}
A_{1} \\
A_{2}
\end{array}\right]^{-1}\left(E \mathscr{W}_{i-1} \oplus E \mathscr{W}_{i-1}\right)
\end{aligned}
$$

with $i \in\{1,2, \ldots, k\}$, where the integer $k \leq n-1$ is determined by the condition $\mathscr{W}_{k+1}=\mathscr{W}_{k}$, i.e., $\mathscr{W}^{\star}=\mathscr{W}_{k}$.

\section{SELF-BOUNDEDNESS}

In this section, we introduce the notion of selfboundedness for implicit 2-D systems. Self-bounded subspaces play a central role in disturbance decoupling problems since they allow such problems to be solved without necessarily making the closed-loop system maximally unobservable [4]. A question that remains open, and which goes beyond the scope of this paper, is wether, as in the 1-D case, solving decoupling problems using self-bounded subspaces ensures maximum assignability of the closed-loop dynamics as proved in the 1-D case in [11]. The concept of self-boundedness can be intuitively introduced as follows. Consider an output-nulling subspace of feedback type $\mathscr{W}$, and a $\mathscr{W}$-valued boundary condition. Is it possible to find a feedback control $u_{i, j}=F x_{i, j}$ such that the partial local state $x_{i, j}$ is not $\mathscr{W}$-valued, but the output continues to remain at zero? Subspaces for which this is not possible are known as self-bounded subspaces.

Definition 2: An output-nulling subspace of feedback type $\mathscr{W}$ is self-bounded if, for any $\mathscr{W}$-valued boundary condition and any control $u_{i, j}=F x_{i, j}$ for which a unique solution $x_{i, j}$ of (1) exists yielding zero output, the partial local state solution of (1) is $\mathscr{W}$-valued, i.e., $x_{i, j} \in \mathscr{W}$ for all $(i, j) \in \mathfrak{B}$.

Notice that in Definition 2 it was necessary to require that the partial local state trajectory that corresponds to zero output be unique, or else the characterisation of self-bounded subspaces where the solution of the system is "trapped" if we insist on maintaining the output at zero becomes meaningless. As a consequence of this, a fundamental difference arises with respect to the notion of self-boundedness in the regular case. Indeed, in the regular case $\mathscr{W}^{\star}$ is trivially self-bounded. In the singular case this is not necessarily the case. In fact, given a control input $u_{i, j}=F x_{i, j}$ where $F$ is a friend of $\mathscr{W}^{\star}$, we know that for every $\mathscr{W}^{\star}$-valued boundary conditions of (1) a solution of 
(1) exists with identically zero output. On the other hand, if $\mathscr{W}^{\star} \cap \operatorname{ker} E \neq\{0\}$, there might exist other solutions to (1) that do not correspond to an identically zero output.

Now, our aim is to provide a geometric characterisation for self-boundedness similar to the one developed in [14] for the regular (and strictly proper) case. In the 1-D strictly proper case, a self-bounded subspace $\mathscr{V}$ of a triple $(A, B, C)$ is defined as an $(A, B)$-controlled invariant subspace contained in the null-space of $C$ such that $\mathscr{V} \supseteq \mathscr{V}^{\star} \cap \operatorname{im} B$, where $\mathscr{V}^{\star}$ represents the largest $(A, B)$ controlled-invariant subspace contained in $\operatorname{ker} C,[1],[2]$. This condition was extended to 1-D singular systems in [6], where a self-bounded subspace $\mathscr{V}$ of a strictly proper system ruled by

$$
\begin{aligned}
E x_{k+1} & =A x_{k}+B u_{k} \\
y_{k} & =C x_{k}
\end{aligned}
$$

is defined an $(E ; A, B)$-controlled invariant subspace (i.e., it satisfies the inclusion $A \mathscr{V} \subseteq E \mathscr{W}+\operatorname{im} B$ ) contained in the null-space of $C$ such that $\bar{E} \mathscr{V} \supseteq E \mathscr{V}^{\star} \cap \operatorname{im} B$. In [13], the definition of self-boundedness was extended to bi-proper systems described by a quadruple $(A, B, C, D)$. In that case, the definition of self-boundedness was given in terms of its system-theoretic property, and an equivalent geometric characterisation was proposed that was characterised by the subspace inclusion $\mathscr{V} \supseteq \mathscr{V}^{\star} \cap B \operatorname{ker} D$. A "natural" way of characterising self-bounded subspaces for 2-D implicit biproper systems is to assume they satisfy the inclusion $E \mathscr{W} \supseteq$ $E \mathscr{W}^{\star} \cap B \operatorname{ker} D$. Unfortunately, we will see that this geometric inclusion is not sufficient to characterise self-bounded subspaces as defined above (indeed, $\mathscr{W}^{\star}$ satisfies this inclusion but as noticed above it is not self-bounded in general). We will show that a correct geometric characterisation of selfboundedness is given by the two conditions

- $\mathscr{W}^{\star} \cap \operatorname{ker} E=\{0\}$

- $E \mathscr{W} \supseteq E \mathscr{W}^{\star} \cap B \operatorname{ker} D$

The first condition was used in a 1-D setting in [6] to identify the case in which the set of output-nulling subspaces satisfying $E \mathscr{W} \supseteq E \mathscr{W}^{\star} \cap \operatorname{im} B$ is closed under intersection, and therefore it admits a minimum, but it was not recognised as being an essential part of the definition of self-boundedness.

The following lemma extends a well-known property for explicit 1-D systems [2, Property 4.1.7].

Lemma 5: Let $F$ be a friend of $\mathscr{W}^{\star}$. Let $\mathscr{W}$ be such that $E \mathscr{W} \supseteq E \mathscr{W}^{\star} \cap B \operatorname{ker} D$. Then, $F$ is a friend of $\mathscr{W}$.

Proof: Since $\mathscr{W} \subseteq \mathscr{W}^{\star}$, there holds

$$
\left[\begin{array}{c}
A_{0}+B F \\
C+D F
\end{array}\right] \mathscr{W} \subseteq\left[\begin{array}{c}
A_{0}+B F \\
C+D F
\end{array}\right] \mathscr{W}^{\star} \subseteq E \mathscr{W}^{\star} \oplus\{0\} .
$$

Moreover, the trivial inclusion $\left[\begin{array}{c}B \\ D\end{array}\right] F \mathscr{W} \subseteq \operatorname{im}\left[\begin{array}{l}B \\ D\end{array}\right]$, together with $\left[\begin{array}{c}A_{0} \\ C\end{array}\right] \mathscr{W} \subseteq E \mathscr{W} \oplus\{0\}+\operatorname{im}\left[\begin{array}{l}B \\ D\end{array}\right]$, gives

$$
\left[\begin{array}{c}
A_{0}+B F \\
C+D F
\end{array}\right] \mathscr{W} \subseteq E \mathscr{W} \oplus\{0\}+\operatorname{im}\left[\begin{array}{c}
B \\
D
\end{array}\right]
$$

The intersection of (8) and (9) and the application of the modular rule ${ }^{1}$ gives

$$
\begin{aligned}
{\left[\begin{array}{c}
A_{0}+B F \\
C+D F
\end{array}\right] \mathscr{W} } & \subseteq(E \mathscr{W} \oplus\{0\})+\left(\left(E \mathscr{W}^{\star} \oplus\{0\}\right) \cap \operatorname{im}\left[\begin{array}{c}
B \\
D
\end{array}\right]\right) \\
& =E \mathscr{W} \oplus\{0\}
\end{aligned}
$$

because $E \mathscr{W} \supseteq E \mathscr{W}^{\star} \cap B \operatorname{ker} D$ is equivalent to $E \mathscr{W} \oplus\{0\} \supseteq$ $\left(E \mathscr{W}^{\star} \oplus\{0\}\right) \cap \operatorname{im}\left[\begin{array}{c}B \\ D\end{array}\right]$.

Differently from explicit 2-D systems, the intersection of two output-nulling subspaces of feedback type satisfying the inclusion $E \mathscr{W} \supseteq E \mathscr{W}^{\star} \cap B \operatorname{ker} D$ does not necessarily satisfy the same subspace inclusion. In other words, given two output-nulling subspaces $\mathscr{W}_{1}$ and $\mathscr{W}_{2}$ of feedback type such that

$$
\begin{aligned}
& E \mathscr{W}_{1} \supseteq E \mathscr{W}^{\star} \cap B \operatorname{ker} D, \\
& E \mathscr{W}_{2} \supseteq E \mathscr{W}^{\star} \cap B \operatorname{ker} D,
\end{aligned}
$$

it is not true in general that

$$
E\left(\mathscr{W}_{1} \cap \mathscr{W}_{2}\right) \supseteq E \mathscr{W}^{\star} \cap B \operatorname{ker} D .
$$

Indeed, in general such intersection $\mathscr{W}_{1} \cap \mathscr{W}_{2}$ may not even give an output-nulling subspace. Consider the following simple example, described by the matrices

$$
\begin{aligned}
E & =\left[\begin{array}{lll}
0 & 1 & 0 \\
0 & 0 & 0
\end{array}\right], \quad A_{0}=\left[\begin{array}{lll}
1 & 1 & 0 \\
0 & 0 & 1
\end{array}\right], \\
A_{1} & =A_{2}=\left[\begin{array}{lll}
0 & 0 & 0 \\
0 & 0 & 0
\end{array}\right], \quad B=\left[\begin{array}{ll}
1 & 1 \\
1 & 0
\end{array}\right], \\
C & =\left[\begin{array}{lll}
0 & 0 & 1
\end{array}\right], \quad D=\left[\begin{array}{ll}
1 & 0
\end{array}\right] .
\end{aligned}
$$

In this case, using the recursion in Lemma 4 we find $\mathscr{W}^{\star}=$ $\mathscr{X}$. It is easy to check that a friend of $\mathscr{W}^{\star}$ is given by the matrix

$$
F=\left[\begin{array}{ccc}
0 & 0 & -1 \\
-\frac{1}{2} & -\frac{1}{2} & \frac{1}{2}
\end{array}\right]
$$

The two subspaces

$$
\mathscr{W}_{1}=\operatorname{span}\left\{\left[\begin{array}{l}
1 \\
1 \\
0
\end{array}\right]\right\} \quad \text { and } \quad \mathscr{W}_{2}=\operatorname{span}\left\{\left[\begin{array}{l}
1 \\
2 \\
0
\end{array}\right]\right\}
$$

are easily seen to be two output-nulling subspaces of feedback type, and they satisfy (10-11). Hence, $F$ is also a friend of $\mathscr{W}_{1}$ and $\mathscr{W}_{2}$. Indeed,

$$
\begin{aligned}
& {\left[\begin{array}{c}
A_{0}+B F \\
C+D F
\end{array}\right] \mathscr{W}_{1}=\operatorname{span}\left\{\left[\begin{array}{l}
1 \\
0 \\
0
\end{array}\right]\right\} \subseteq E \mathscr{W}_{1} \oplus\{0\}} \\
& {\left[\begin{array}{c}
A_{0}+B F \\
C+D F
\end{array}\right] \mathscr{W}_{2}=\operatorname{span}\left\{\left[\begin{array}{l}
1 \\
0 \\
0
\end{array}\right]\right\} \subseteq E \mathscr{W}_{2} \oplus\{0\}}
\end{aligned}
$$

${ }^{1}$ Given three subspaces $\mathscr{X}, \mathscr{Y}, \mathscr{Z}$ of the same vector space, there holds

$$
\begin{aligned}
& \mathscr{X} \cap(\mathscr{Y}+\mathscr{Z}) \subseteq(\mathscr{X} \cap \mathscr{Y})+(\mathscr{X} \cap \mathscr{Z}) \\
& \mathscr{X}+(\mathscr{Y} \cap \mathscr{Z}) \subseteq(\mathscr{X}+\mathscr{Y}) \cap(\mathscr{X}+\mathscr{Z}) .
\end{aligned}
$$

The modular rule says that these inclusions hold with the equality sign if any one of the involved subspaces $\mathscr{X}, \mathscr{Y}, \mathscr{Z}$ is contained in any of the others. 
However, the intersection $\mathscr{W}=\mathscr{W}_{1} \cap \mathscr{W}_{2}=\{0\}$ is not self-bounded. Indeed, while $E \mathscr{W}=\{0\}$, we find $E \mathscr{W}^{\star} \cap$ $B \operatorname{ker} D=\operatorname{span}\left\{\left[\begin{array}{l}1 \\ 0\end{array}\right]\right\}$.

The next theorem, whose proof is omitted, introduces a geometric characterisation of self-boundedness along the same lines of the classic definition given in [1] for regular 1-D systems.

Theorem 3: The output-nulling subspace of feedback type $\mathscr{W}$ is self-bounded if and only if

$$
\begin{array}{r}
\mathscr{W}^{\star} \cap \operatorname{ker} E=\{0\} \\
E \mathscr{W} \supseteq E^{\star} \cap B \operatorname{ker} D
\end{array}
$$

We denote by $\Phi(\Sigma)$ the set of self-bounded subspaces of $\Sigma$, i.e.,

$\Phi(\Sigma) \stackrel{\text { def }}{=}\left\{\mathscr{W} \in \mathscr{W}(\Sigma) \mid E \mathscr{W} \supseteq E \mathscr{W}^{\star} \cap B \operatorname{ker} D, \mathscr{W}^{\star} \cap \operatorname{ker} E=\{0\}\right\}$

We now generalise an important result [2, Property 4.1.8] of self-boundedness that is essential to establish that $(\Phi(\Sigma),+, \cap ; \subseteq)$ is a lattice.

Lemma 6: The intersection of two self-bounded subspaces of feedback type is self-bounded of feedback type.

Proof: Let $\mathscr{W}_{1}$ and $\mathscr{W}_{2}$ be two self-bounded subspaces of feedback type. Let $\mathscr{W}=\mathscr{W}_{1} \cap \mathscr{W}_{2}$. Consider a friend $F$ of $\mathscr{W}^{\star}$. From Lemma 5, we find

$$
\left[\begin{array}{c}
A_{0}+B F \\
C+D F
\end{array}\right] \mathscr{W}_{i} \subseteq E \mathscr{W}_{i} \oplus\{0\}, \quad i \in\{1,2\} .
$$

Thus,

$$
\begin{aligned}
{\left[\begin{array}{c}
A_{0}+B F \\
C+D F
\end{array}\right] \mathscr{W} } & \subseteq\left[\begin{array}{c}
A_{0}+B F \\
C+D F
\end{array}\right] \mathscr{W}_{1} \cap\left[\begin{array}{c}
A_{0}+B F \\
C+D F
\end{array}\right] \mathscr{W}_{2} \\
& \subseteq\left(E \mathscr{W}_{1} \oplus\{0\}\right) \cap\left(E \mathscr{W}_{2} \oplus\{0\}\right) \\
& =\left(E \mathscr{W}_{1} \cap E \mathscr{W}_{2}\right) \oplus\{0\} \\
& =E\left(\mathscr{W}_{1} \cap \mathscr{W}_{2}\right) \oplus\{0\}
\end{aligned}
$$

where the last equality follows from the fact that $\mathscr{W}^{\star} \cap$ $\operatorname{ker} E=\{0\}$. In fact, in general there holds $E\left(\mathscr{W}_{1} \cap \mathscr{W}_{2}\right) \subseteq$ $E \mathscr{W}_{1} \cap E \mathscr{W}_{2}$. In this case, however, such relation holds with the equality sign. Indeed, let $\xi \in E \mathscr{W}_{1} \cap E \mathscr{W}_{2}$. This means that two vectors $\xi_{1} \in \mathscr{W}_{1}$ and $\xi_{2} \in \mathscr{W}_{2}$ exist such that $\xi=E \xi_{1}=E \xi_{2}$. This implies that the difference $\xi_{1}-\xi_{2}$ is a vector of $\operatorname{ker} E$. On the other hand, such difference is also an element of $\mathscr{W}^{\star}$, since $\mathscr{W}_{1} \subseteq \mathscr{W}^{\star}$ and $\mathscr{W}_{2} \subseteq \mathscr{W}^{\star}$. Since $\mathscr{W}^{\star} \cap \operatorname{ker} E=\{0\}$, we find that $\bar{\xi}_{1}=\xi_{2}$, and therefore $\xi \in E\left(\mathscr{W}_{1} \cap \mathscr{W}_{2}\right)$. By intersecting $E \mathscr{W}_{i} \supseteq E \mathscr{W}^{\star} \cap B \operatorname{ker} D$ for $i \in\{1,2\}$, we get $E \mathscr{W}_{1} \cap E \mathscr{W}_{2} \supseteq E \mathscr{W}^{\star} \cap B \operatorname{ker} D$. Since as aforementioned $\mathscr{W}^{\star} \cap \operatorname{ker} E=\{0\}$ guarantees that $E \mathscr{W}_{1} \cap$ $E \mathscr{W}_{2}=E\left(\mathscr{W}_{1} \cap \mathscr{W}_{2}\right)$, we find $E\left(\mathscr{W}_{1} \cap \mathscr{W}_{2}\right) \supseteq E \mathscr{W}^{\star} \cap B \operatorname{ker} D$, which implies that $\mathscr{W}$ is self-bounded of feedback type.

Theorem 4: Let $\mathscr{W}^{\star} \cap \operatorname{ker} E=\{0\}$. Let $F$ be a friend of $\mathscr{W}^{\star}$. The minimum element of the lattice $(\Phi(\Sigma),+, \cap ; \subseteq)$ is given by the last term $\mathscr{R}^{\star}$ of the monotonically nondecreasing sequence of subspaces $\left\{\mathscr{R}_{i}\right\}_{i}$ given by

$$
\begin{aligned}
& \left\{\begin{array}{l}
\mathscr{R}_{0}=\mathscr{W}^{\star} \cap E^{-1} B \operatorname{ker} D \\
\mathscr{R}_{i}=\mathscr{R}_{i-1}+\left(E^{-1}\left(A_{0}+B F\right) \mathscr{R}_{i-1} \cap \mathscr{W}^{\star}\right)
\end{array}\right. \\
& +\sum_{j=1}^{2}\left(E^{-1} A_{j} \mathscr{R}_{i-1} \cap \mathscr{W}^{\star}\right),
\end{aligned}
$$

with $i \in\{1,2, \ldots, k\}$, where the integer $k \leq n-1$ is determined by the condition $\mathscr{R}_{k+1}=\mathscr{R}_{k}$, i.e., $\mathscr{R}^{\star}=\mathscr{R}_{k}$.

Notice that subspace $\mathscr{R}^{\star}$, as defined above, is the smallest $\left(E ; A_{0}+B F, A_{1}, A_{2}\right)$ invariant subspace of $\mathscr{X}$ that contains the subspace $\mathscr{W}^{\star} \cap E^{-1} B \operatorname{ker} D$. The proof can be carried out by adapting the proof of [14, Lemma 7.10] to the argument used in the proof of [6, Lemma 3.1]. In particular, i) $\mathscr{R}^{\star}$ is output-nulling; ii) $\mathscr{R}^{\star}$ is self-bounded; iii) $\mathscr{R}^{\star}$ does not depend on the particular choice of the friend $F$ of $\mathscr{W}^{\star} ; i v$ ) $\mathscr{R}^{\star}$ is the smallest among all the self-bounded subspaces of $\Sigma$. It is also worth noticing that, unlike the 1-D case, $\mathscr{R}^{\star}$ does not coincide with the intersection of the largest output-nulling subspace of feedback type with the smallest input-containing subspace of output-injection type, as it was proved (in the regular strictly proper case) in [14, p. 352].

\section{Duality And SElF-Hiddenness}

The dual concept of 2-D controlled invariance is called 2-D conditioned invariance. While 2-D controlled invariant subspaces reside in the state-space $\mathscr{X}$, their duals lie in the outer state space $\underline{\mathscr{X}}$. A subspace $\underline{\mathscr{S}}$ of the outer space $\underline{\mathscr{X}}$ is conditioned invariant for $\Sigma$ if

$$
A_{i}\left(E^{-1} \underline{\mathscr{S}} \cap \operatorname{ker} C\right) \subseteq \underline{\mathscr{S}}, \quad i \in\{0,1,2\},
$$

see [15]-[16]. The duality between 2-D controlled and conditioned invariance can be stated in precise terms as follows. Let $\Sigma^{\mathrm{T}}$ identify the dual system of $(1-2)$, i.e., $\Sigma^{\mathrm{T}} \stackrel{\text { def }}{=}$ $\left(E^{\mathrm{T}} ; A_{0}^{\mathrm{T}}, A_{1}^{\mathrm{T}}, A_{2}^{\mathrm{T}} ; C^{\mathrm{T}} ; B^{\mathrm{T}} ; D^{\mathrm{T}}\right)$. Then, the orthogonal complement of a controlled invariant for $\Sigma$ is conditioned invariant for $\Sigma^{\mathrm{T}}$, and vice-versa. The duals of 2-D output-nulling subspaces are the 2-D input-containing subspaces, which can be defined as the subspaces $\mathscr{\mathscr { S }}$ satisfying the subspace inclusion

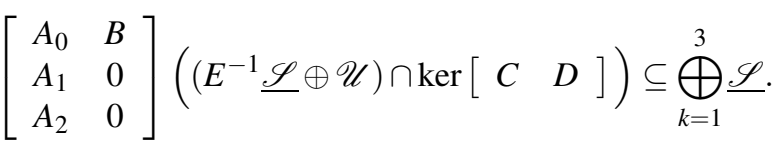

The set of input-containing subspaces of $\Sigma$ is denoted by $\mathscr{S}(\Sigma)$. This set is closed under subspace intersection but not under subspace addition. Therefore, $(\mathscr{S}(\Sigma), \cap ; \subseteq$ ) is a (nondistributive and modular) lower semilattice with respect to the binary operation $\cap$ and with respect to the partial ordering $\subseteq$. Thus, it admits a minimum given by $\mathscr{S}^{\star}=\min \mathscr{S}(\Sigma)=$ $\bigcap_{\mathscr{S} \in \mathscr{S}(\Sigma)} \mathscr{S}$. By dualising the algorithm for $\mathscr{V}^{\star}$, we have the following.

Lemma 7: [15]-[16]. $\mathscr{S}^{\star}$ is the last term of the monotonically non-decreasing sequence of subspaces $\left\{\mathscr{S}_{i}\right\}_{i}$ given by

$$
\left\{\begin{aligned}
\mathscr{\mathscr { S }}_{0} & =0 \\
\underline{\mathscr{S}}_{i} & =\sum_{j=1}^{2}\left[\begin{array}{ll}
A_{j} & O
\end{array}\right]\left(\left(E^{-1} \underline{\mathscr{S}}_{i-1} \oplus \mathscr{U}\right) \cap \operatorname{ker}\left[\begin{array}{ll}
C & D
\end{array}\right]\right) \\
& +\left[\begin{array}{ll}
A_{0} & B
\end{array}\right]\left(\left(E^{-1} \underline{\mathscr{S}}_{i-1} \oplus \mathscr{U}\right) \cap \operatorname{ker}\left[\begin{array}{ll}
C & D
\end{array}\right]\right)
\end{aligned}\right.
$$


where the integer $k \leq n-1$ is determined by the condition $\mathscr{S}_{k+1}=\underline{\mathscr{S}}_{k}$, i.e., $\underline{\mathscr{S}}_{k}=\underline{\mathscr{S}}^{\star}$.

The dual of controlled invariance of feedback type was introduced in [15]-[16] with the name of conditioned invariance of output-injection type. A subspace $\underline{Z}$ is conditioned invariant of output-injection type for $\Sigma$ if

- $A_{0}\left(E^{-1} \underline{\mathscr{Z}} \cap \operatorname{ker} C\right) \subseteq \underline{Z}$;

- $A_{1} E^{-1} \underline{\mathscr{Z}} \cap A_{2} E^{-1} \underline{\mathscr{Z}} \subseteq \underline{\mathscr{Z}}$.

For conditioned invariant subspaces of output-injection type the existence of a matrix $G$ is guaranteed such that

$\left(A_{0}+G C\right) E^{-1} \underline{\mathscr{Z}} \subseteq \underline{\mathscr{Z}}, \quad A_{1} E^{-1} \underline{\mathscr{Z}} \subseteq \underline{\mathscr{Z}}, \quad A_{2} E^{-1} \underline{\mathscr{Z}} \subseteq \underline{\mathscr{Z}}$.

Conditioned invariant subspaces of output-injection type and controlled invariant subspaces of feedback type are dual objects. In order to define a notion of self-hiddenness for $\Sigma$, we also need to define input-containing subspaces of outputinjection type. A subspace $\underline{\mathscr{Z}}$ is an input-containing subspace of output-injection type for $\Sigma$ if

$$
\begin{aligned}
& {\left[\begin{array}{ll}
A_{0} & B
\end{array}\right]\left(\left(E^{-1} \underline{\mathscr{Z}} \oplus \mathscr{U}\right) \cap \operatorname{ker}\left[\begin{array}{ll}
C & D
\end{array}\right]\right) \subseteq \underline{\mathscr{Z}}} \\
& A_{i} E^{-1} \underline{\mathscr{Z}} \subseteq \underline{\mathscr{Z}} \quad i \in\{1,2\} .
\end{aligned}
$$

Input-containing subspaces of output-injection type are the duals of output-nulling subspaces of feedback type. It follows that the set of input-containing subspaces of outputinjection type $\mathscr{Z}(\Sigma)$ is closed under intersection but not under addition, and $(\underline{Z}(\Sigma), \cap ; \subseteq)$ is a lower semilattice, whose minimum is denoted by $\underline{\mathcal{Z}}^{\star}$, which can be obtained by dualising the algorithm in Lemma 4 :

$$
\left\{\begin{aligned}
\underline{Z}_{0} & =0 \\
\mathscr{Z}_{i} & =\left[\begin{array}{ll}
A_{0} & B
\end{array}\right]\left(\left(E^{-1} \underline{\mathscr{Z}}_{i-1} \oplus \mathscr{U}\right) \cap \operatorname{ker}\left[\begin{array}{ll}
C & D
\end{array}\right]\right) \\
& +\left[\begin{array}{ll}
A_{1} & A_{2}
\end{array}\right]\left(E^{-1} \underline{\mathscr{Z}}_{i-1} \oplus E^{-1} \underline{\mathscr{Z}}_{i-1}\right)
\end{aligned}\right.
$$

A self-hidden subspace $\underline{\mathscr{Z}}$ for $\Sigma$ is an input-containing subspace of output-injection type for $\Sigma$ which satisfies

$$
\begin{aligned}
& E^{-1} \underline{\mathscr{Z}} \subseteq E^{-1} \frac{\mathscr{Z}^{\star}}{\mathscr{X}}+C^{-1} \operatorname{im} D, \\
& \underline{\mathscr{Z}}^{\star}+\operatorname{im} E=\underline{\mathscr{X}} .
\end{aligned}
$$

The dual of a self-hidden subspace is a self-bounded subspace. We denote by $\Psi(\Sigma)$ the set of self-hidden subspaces of $\Sigma$ :

$$
\begin{gathered}
\Psi(\Sigma) \stackrel{\text { def }}{=}\left\{\underline{\mathscr{Z}} \in \underline{\mathscr{Z}}(\Sigma) \mid E^{-1} \underline{\mathscr{Z}} \subseteq E^{-1} \underline{\mathscr{Z}^{\star}}+C^{-1} \mathrm{im} D,\right. \\
\left.\underline{\mathscr{Z}}^{\star}+\mathrm{im} E=\underline{\mathscr{X}}\right\}
\end{gathered}
$$

As a result of this duality, it follows that $\Psi(\Sigma)$ is also a lattice. Its minimum element is $\underline{\mathscr{Z}}^{\star}$. Its largest element can be obtained by dualising the algorithm in Theorem 4 as

$$
\begin{aligned}
& \left\{\begin{array}{l}
\underline{\mathscr{Q}}_{0}=\underline{\mathscr{Z}}^{\star}+E C^{-1} \mathrm{im} D \\
\underline{\mathscr{Q}}_{i}=\underline{\mathscr{Q}}_{i-1} \cap\left(E\left(A_{0}+G C\right)^{-1} \underline{\mathscr{Q}}_{i-1}+\underline{\mathscr{Z}}^{\star}\right)
\end{array}\right. \\
& \cap \bigcap_{j=1}^{2}\left(E A_{j}^{-1} \underline{\mathscr{Q}}_{i-1}+\underline{\mathscr{Z}}^{\star}\right),
\end{aligned}
$$

where $G$ is a friend of $\underline{\mathscr{Z}}^{\star}$, i.e., it satisfies

$$
\left[\begin{array}{cc}
A_{0}+G C & B+G D \\
A_{1} & 0 \\
A_{2} & 0
\end{array}\right]\left(E^{-1} \underline{\mathscr{Z}^{\star}} \oplus \mathscr{U}\right) \subseteq \bigoplus_{k=1}^{3} \underline{\mathscr{Z}}^{\star} .
$$

The sequence is monotonically non-increasing, and converges in at most $n-1$ steps to the subspace $\underline{\mathscr{Q}}^{\star}=\min \Psi(\Sigma)$.

\section{CONCLUSIONS}

In this paper we have introduced the concepts of self-boundedness and self-hiddenness for 2-D implicit Fornasini-Marchesini models. We have observed that the geometric characterisation of these subspaces within this context is richer than in the case of regular 2-D models.

\section{REFERENCES}

[1] G. Basile and G. Marro, "Self-bounded controlled invariant subspaces: a straightforward approach to constrained controllability," J. Optim. Theory and Applic., 38(1), pp. 71-81, 1982.

[2] G. Basile and G. Marro, Controlled and Conditioned Invariants in Linear System Theory. Prentice Hall, New Jersey, 1992.

[3] P. Bernhard. "On singular implicit linear dynamical systems," SIAM J. Control and Optimization, 20(5):612-633, 1982.

[4] G. Conte and A. Perdon. A geometric approach to the theory of 2-D systems. IEEE Trans. Aut. Contr., AC-33:946-950, 1988.

[5] G. Conte, A. Perdon, and T. Kaczorek. Geometric methods in the theory of singular 2D linear systems. Kybernetika, vol. 27:263-270, 1991.

[6] M.L. Corradini. Self bounded controlled invariants for singular systems. Kybernetika, vol. 30:639-644, 1994.

[7] T. Kaczorek. Singular general model of 2D systems and its solutions. IEEE Trans. Aut. Contr., AC-33:1060-1061, 1988.

[8] E. Fornasini and G. Marchesini, "State-space realization theory of twodimensional filters," IEEE Trans. Aut. Contr., AC-21, no. 4, pp. 484492, August 1976.

[9] E. Fornasini and G. Marchesini, "Doubly-Indexed Dynamical Systems: State-Space Models and Structural Properties," Math. Syst. Theory, vol. 12, pp. 59-72, 1978.

[10] A. Karamanciog̃lu and F.L. Lewis. Geometric theory for the singular Roesser model. IEEE Trans. Autom. Contr., AC-37(6):801-806, June 1992.

[11] M. Malabre, J. Martínez-García, and B. Del-Muro-Cuéllar, "On the fixed poles for disturbance rejection," Automatica, vol. 33, no. 6, pp. 1209-1211, 1997.

[12] A. Morse, "Structural invariants of linear multivariable systems," SIAM J. Contr., 11(3), pp. 446-465, 1973.

[13] L. Ntogramatzidis, "Self-bounded output-nulling subspaces for non strictly proper systems and their application to the disturbance decoupling problem". IEEE Trans. Aut. Contr., vol. 53, no. 1, pp. 423-428, 2008.

[14] L. Ntogramatzidis, "Structural invariants of two-dimensional systems". SIAM Journal on Control and Optimization, 50(1): 334-356, 2012.

[15] L. Ntogramatzidis, and M. Cantoni. "Structural invariants for implicit two-dimensional systems". In Proceedings of the $7^{\text {th }}$ International Workshop on Multidimensional (nD) Systems (NdS 2011), Poitiers, France, September 5-7, 2011.

[16] L. Ntogramatzidis, and M. Cantoni, "Geometric techniques for implicit two-dimensional systems". Multidimensional Systems and Signal Processing, Accepted for publication.

[17] L. Ntogramatzidis, M. Cantoni and R. Yang, "On the partial realisation of non-causal 2-D linear systems". IEEE Transactions on Circuits and Systems - I, 54(8): 1800-1808, 2007.

[18] L. Ntogramatzidis, M. Cantoni, and R. Yang, "A geometric theory for 2-D systems including notions of stabilisability". Multidim. Syst. Signal Proc., 19: 449-475, 2008.

[19] L. Ntogramatzidis, and M. Cantoni, "Detectability subspaces and observer synthesis for two-dimensional systems", Multidim. Syst. Signal Proc., In Press. Available on-line.

[20] H.L. Trentelman, A.A. Stoorvogel, and M. Hautus. Control theory for linear systems. Communications and Control Engineering. Springer, 2001.

[21] W.M. Wonham. Linear Multivariable Control: A Geometric Approach. Springer-Verlag, 3 edition, 1985. 